\title{
Co-FATORES DO HPV NA ONCOGÊNESE CERVICAL
}

\author{
Álvaro P. Pinto*, Siumara Tulio, Olívia Russo Cruz \\ Trabalho realizado no Serviço de Anatomia Patológica do Hospital de Clínicas da UFPR \\ e Laboratório de Citopatologia, Anatomia Patológica e Patologia Molecular ANNALAB, Curitiba, PR.
}

RESUMO - O papilomavírus humano (HPV) exerce um papel central na carcinogênese do colo uterino. Em torno a ele orbitam outros fatores que influenciam direta ou indiretamente a instalação deste mecanismo no epitélio escamoso cervical. Investigações a respeito dos mecanismos de atuação e interação desses co-fatores com os elementos virais encontram-se na literatura dos últimos 20 anos. 0 presente artigo de revisão explora os possíveis co-fatores do HPV na gênese do carcinoma escamoso do colo uterino, levando em conta apenas os fatores cuja associação com o vírus ou câncer cervical tenha sido documentada experimentalmente, e não apenas clínica ou epidemiologicamente. Dentre os parâmetros abordados estão os fatores imunológicos (resposta imune local e humoral), a associação com a Síndrome da Imunodeficiência Adquirida Humana, fatores genéticos como o polimorfismo da proteína p53, o tabagismo e o uso de contraceptivos orais. Todos estes fatores interagem em menor ou maior intensidade com oncoproteínas e outros elementos do HPV, potencializando a ação do vírus na célula hospedeira e facilitando o desenvolvimento dos processos de imortalização e carcinogênese.

Unitermos: Papilomavirus. Câncer cervical. Imunossupressão. Fumo. Contraceptivos orais.

\section{INTRODUÇÃO}

Após mais de 20 anos de investigações sobre a associação existente entre 0 papilomavírus humano (HPV) e o carcinoma escamoso cervical, poucas dúvidas nos restam a respeito do papel central deste vírus na carcinogênese cervical ${ }^{52}$. Apesar deste assunto estar longe de ser totalmente elucidado, o foco das atenções passa hoje para o desenvolvimento de vacinas para 0 vírus e a prevenção da instalaçãa do processo oncogênico cervical ${ }^{5,25}$. Paralelamente, avançam as investigações a respeito dos possíveis co-fatores do HPV e o entendimento de seus mecanismos de atuação e interação com os elementos virais. O presente artigo de revisão parte do princípio que o HPV é o principal fator causal do carcinoma escamoso cervical. Este fato é comprovado pelas evidencias da literatura

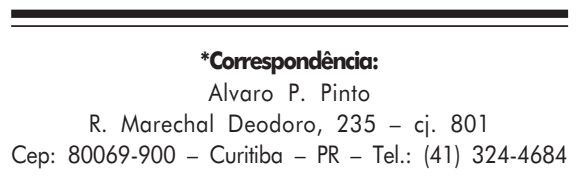

dos últimos 30 anos $2,6,16,34,36,40,42,47,52,55$, bem como pelo esforço recente de pesquisadores no desenvolvimento de vacinas contra este vírus, com a finalidade de prevenção deste tipo de câncer ${ }^{26,30,45,52}$. A partir daí, esta revisão passa a explorar os possíveis co-fatores do HPV no processo de carcinogênese, levando em conta apenas os fatores cuja a associação com o vírus ou câncer cervical tenha sido documentada experimentalmente, e não apenas clínica ou epidemiologicamente. Por não ser 0 objetivo desta revisão, fatores que têm sido vinculados ao HPV por estudos puramente epidemiológicos não serão abordados. Dentre eles incluem-se o início precoce da atividade sexual, multiplicidade de parceiros, baixa escolaridade e renda, multiparidade e história de doenças sexualmente transmissíveis ${ }^{8}$, incluindo infecção por clamídia 33,29 . O presente trabalho visa atualizar o leitor a respeito das certezas e incertezas da literatura acerca dos principais cofatores do HPV na gênese do carcinoma escamoso de colo uterino.

\section{Fatores imunológicos}

Vários estudos na literatura suportam a forte associação existente entre a oncogênese e progressão neoplásica relacionada ao HPV e ao sistema imunológico. Entretanto, os mecanismos exatos que disparam o gatilho de uma resposta imune eficiente contra lesões relacionadas ao HPV ainda não são conhecidos. Eles podem estar relacionados à ativação do sistema imunológico ou à composição genética do hospedeiro.

Umavez que a exposição direta das oncoproteínas do HPV a células imunocompetentes não ocorre, é concebível que o desenvolvimento de anticorpos às proteínas virais possa depender de uma infecção secundária por meio de pequenas abrasões ou soluções de continuidade do epitélio, resultando na exposição das proteínas virais a estas células. No câncer relacionado ao HPV, este mecanismo depende da ocorrência de necrose celular do tecido proliferante invasor ${ }^{18}$.

\section{Resposta imune local}

A presença de células monomorfonucleares, principalmente células CD4 e ma- 
Pinto AP L ET AL.

crófagos, tem sido demonstrada em papilomas em regressão ${ }^{48}$. O papel da resposta imune local na carcinogênese cervical foi quantitativamente estudado por imunohistoquímica ${ }^{49}$. Uma associação significativa entre o aumento do número de células imunocompetentes e o aumento do grau da neoplasia intra-epitelial cervical (NIC)foi encontrado. Este achado indica que a resposta imune local pode ser importante para o desenvolvimento e progressão do câncer cervical, principalmente em um estágio inicial do processo carcinogênico ${ }^{49}$. Em contrapartida, uma diminuiçãa do número de células de Langerhans na neoplasia intra-epitelial grau I (NIC I) em fumantes e mulheres grávidas foi verificada por outro estudo ${ }^{43}$. Achados similares foram também demonstrados previamente por outros autores e podem ser um indício da interação existente entre elementos imunológicos e agentes como o HPV, hormônios esteróides e o fumo, na etiologia da neoplasia cervical ${ }^{4}$.

\section{Resposta imune humoral}

O papel da resposta imune humoral no controle da infecção por HPV e lesões relacionadas está também distante de ser completamente compreendido, entretanto, ela parece ser capaz de impedir a infecção pelo papilomavírus. Associações entre anticorpos contra proteínas do HPV e doenças relacionadas ao vírus foram estabelecidas pordiversos estudos na literatura ${ }^{58}$. Amaioria destes estudos basearam-se em antígenos sintéticos ou recombinantes que assemeIham-se, na função e imunogenicidade, às proteínas autênticas de expressão tardia (não estruturais) e precoce (estruturais) do HPV, e foram detectados em diversos experimentos, incluindo ELISA, Western blotting e mais recentemente imunoprecipitação. Dentre os antígenos utilizados nestes estudos, proteínas recombinantes de expressão tardia do HPV, que compõem o capsídeo do vírus, denominadas partículas vírus-seme-
Ihantes (VLPs), têm sido as mais documentadas. Apesar de sua sensibilidade relativamente baixa, estes estudos têm encontrado anticorpos aos capsídeos do vírus (mensurados por meio do uso de VLPs) e a algumas proteínas virais de expressão precoce em uma proporção significativa de paciente com doenças benignas e malignas relacionadas ao HPV. Um número pequeno de pacientes, entretanto, é desprovido de anticorpos detectáveis, mesmo quando testados por uma combinação de métodos que aumentaria o valor preditivo do teste sorológico. Este fato pode estar relacionado a diferenças na apresentação do antígeno durante o curso da infecçã̃o ou mesmo a outro fator de significado biológico desconhecido, ainda a ser esclarecido por estudos futuros.

\section{Associação com a Síndrome da Imu- nodeficiência Humana}

Mulheres imunodeprimidas apresentam risco elevado para o desenvolvimento de neoplasiaescamosa intra-epitelial e invasiva do trato genital inferior. Isto inclui pacientes que foram submetidas a transplantes de órgãos e estão sob medicação imunossupressiva, têm doença de Hodgkin ou estão infectadas pelo $H_{I V}{ }^{18}$. O elo entre doenças relacionadas ao HPV e esta última categoria é ainda mais forte, porque ambas condições, as infecções por HPV e HIV, são transmitidas sexualmente e suas populações de risco apresentam várias características demográficas em comum. De outro modo, estudos do tipo caso-controle têm demonstrado que apesar destas associações, infecção por HPV e soropositividade para HIV, serem fatores de risco independentes para o desenvolvimento de NIC, a soropositividade para HIV é um fator de risco independente para infecções por HPV, nas formas latente e clínica. Os mesmos estudos têm demonstrado que um baixo número de linfócitos T CD4+ (menos de 200 células $/ \mathrm{ml}$ ) está também especificamente associado com infecções por
HPV e o desenvolvimento de NIC bem como que a proporção entre infecções latentes e clínicas pelo HPV eleva-se com o aumento do número de linfócitos T CD4+ de menos de 200 células/ml para níveis normais em pacientes HIV negativos. Todavia, se HPV e HIV estão diretamente ou indiretamente associados à imunossupressão relacionada ao vírus HIV permanece obscuro. Uma vez que os dois vírus não co-infectam o colo uterino, se interações moleculares ocorrem entre o HIV e o HPV, elas são provavelmente mediadas por fatores extra-celulares. $O$ aumento da expressão de genes do HPV em mulheres infectadas pelo HIV pode potencialmente ser explicada por interações envolvendo a proteína transativatora do HIV-I (tat I) e a proteína promotora do HPV 16 (p97), levando a reversão da repressão de E2 (Figura I). De outra maneira, a mesma proteína do HIV temtambém demonstrado ser capaz de inibir a proliferação de células $T$ in vitro.

\section{Polimorfismo da proteína p53}

De acordo com Lo et al (1992) $)^{31}$, a mutação do gene que codifica a proteína 53 (p53) não é um acontecimento comum no câncer cervical, ocorrendo em 3 dos 14 casos estudados por estes autores. Entretanto, ela pode estar relacionada com a carcinogênese pelo HPV segundo outros autores $^{31,58}$. Estes mesmos autores demonstraram que a suscetibilidade da p53 ao HPV está relacionada com seu genótipo. 0 polimorfismo no códon 72 do gene codificante pode resultar na produção de uma proteína com unidades dos aminoácidos Prolina (Pro) ou Arginina (Arg). Em 1998, Story et al demonstraram que mulheres homozigotas para Arg eram sete vezes mais suscetíveis ao carcinoma cervical do que as heterozigotas, Arg-Pro'. Posteriormente, em um estudo realizado com mulheres italianas e suíças, Zehle et al. (1999) comprovaram que as pacientes apresentando 


\section{Figura I - Esquema representativo do genoma do HPV - I6}

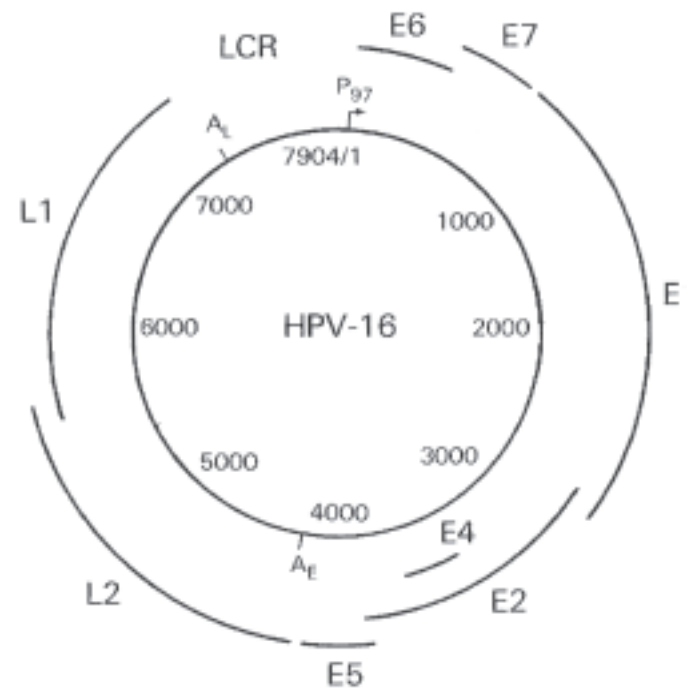

homozigose para Arg tinham maior risco para o carcinoma ${ }^{59}$. No entanto, pesquisas desenvolvidas no Japão, Europa e Estados Unidos não têm demonstrado esta mesma associaçãa ${ }^{32,17,51}$. Recentemente, Agorastos et al. (2000) confirmaram os resultados obtidos por Story (1998) e observaram que tanto o grupo étnico como os métodos laboratoriais utilizados, o grau da lesão por HPV e o tamanho da amostra podem ser os responsáveis pelos resultados controversos anteriormente publicados'. Apesar da controvérsia, evidências levam a crer que a homozigose paraArg pode ser uma característica genética de alguma importância parao desenvolvimento da NIC.

\section{Tabagismo}

Exposição, idade de início, período e freqüência de consumo de cigarros, todos estes fatores parecem influenciar na incidência de NIC e câncer cervical21,22,28. Os dois mecanismos principais pelo qual o hábito de fumar contribui para a oncogênese cervical incluem a exposição direta do DNA de células epiteliais cervicais a nicotina e a cotidina, e a produtos metabólicos do tipo esperado a partir de reações com hidrocarbonetos policíclicos aromáticos e aminas aromáticas, outros componentes da fumaça do cigarro ${ }^{23,46}$. As associações entre diferentes genótipos de enzimas carcinógenasmetabolizantes, fumo e neoplasia cervical foram investigadas por dois estudos de Warwick etal. ${ }^{1,56,57}$. O primeiro estudo deste autor concluiu que fumantes com o genótipo tipo selvagem da enzima detoxificante citocromo P450 fase I (CYP2D6), genótipo metabolizador extensivo (EM), têm um risco aumentado para $\mathrm{NIC} \mathrm{I//II,} \mathrm{mas} \mathrm{um} \mathrm{risco}$ reduzido para carcinoma escamoso cervical invasivo ${ }^{13}$. Este achado corrobora com ofato de que certas $\mathrm{N}$-nitrosaminas específicas do tabaco são substrato para CYP2D6, e por esta razão podem estar envolvidas na patogênese da $\mathrm{NIC}^{13}$. De qualquer modo, os carcinógenos necessários para a progressão para neoplasia invasora e presumidamente efetivamente degradados por CYP2D6-EM ainda não são conhecidos. O segundo estudo realizado por Warwick et al. não encontrou associação significativa entre outro genótipo da S-transferase classe theta glutationa (GSTTI) e a neoplasia cervical, da mesma forma do que foi verificado anteriormente com o genótipo S-transferase glutationa fase 2 (GSTMI), por este mesmo autor $r^{66,57}$.

Um segundo mecanismo que pode explicar a carcinogênese relacionada ao tabaco é a imunosupressão. Alterações verificadas no sistema imune periférico de pacientes fumantes incluem a elevação do número de células sangüíneas, o aumento do número de linfócitos T citotóxicos/supressores, a diminuição do número de linfócitos $T$ indutores/auxiliares, discreta supressão da atividade de linfócitos $T$, significativo decréscimo da atividade de linfócitos natural killer, e baixos níveis sangüíneos de imunoglobulinas, exceto pela IgE, a qual é elevada ${ }^{27}$. Uma diminuição do número de células de Langerhans na cervix de mulheres fumantes tem sido observada por muitos autores ${ }^{43,50}$. Este dado experimental é apoiado por estudos clínicos que revelam a associação do hábito de fumar e história de condições immunosupressivas em pacientes jovens com doença invasiva cervical ${ }^{43}$.

\section{Contraceptivos orais}

Diferente de outras neoplasias malignas ginecológicas de alta prevalência, como o carcinoma ductal mamário e o adenocarcinoma de endométrio, o câncer escamoso cervical tradicionalmente não tem sido considerado como hormônio-dependente. Entretanto, hormônios esteróides na forma de contraceptivos comumente administrados durante a fase reprodutiva parecem aumentar a atividade transformadora dos oncogenes do HPV e interferir na resolução eficiente de lesões causadas pelo vírus na cérvix de mulheres jovens ${ }^{28,22}$. 
PINTO AP L ET AL.

Monsonego ${ }^{39}$ demonstrou a presença de altos níveis de receptores hormonais (HR), particularmente receptores de progesterona (PR), em NIC I e $\mathrm{NIC} \mathrm{II/III.} \mathrm{Neste} \mathrm{estudo,} \mathrm{foram} \mathrm{localiza-}$ dos PR nos núcleos de fibroblastos estromais subjacentes ao epitélio displásico, sugerindo que este hormônio possa atuar indiretamente, in vivo, em células epiteliais infectadas pelo HPV. Receptores de glicocorticóides", dentre outros fatores celulares de transcrição, têm sido identificados como capazes de ligarem-se à região regulatória upstream de vários HPVs e aumentar o nível de expressão dos oncogenes virais E6 e E7, oncoproteínas capazes de induzir defeitos mitóticos ${ }^{14}$ (Figura 2). Hormônios glicocorticóides parecem regular uma proteína potencializadora de E2 na região URR do HPVI6, em células de carcinoma cervical ${ }^{20}$. Dois elementos responsivos a glucocorticóides (GRE) também são conhecidos por mediar uma resposta a progesterona . A ativação de URR por progesterona e a inibição por anti-progesterona RU 486 têm sido demostradas in vitro ${ }^{36}$. Há ainda especulação a respeito da existência de um elemento responsivo a estrógeno (ERE) baseada na ativação de URR do HPVI6 por estrógeno em células $\mathrm{SiHa}^{37}$. Recentemente, a ativação de transcrição da região regulatória upstream (URR) foi demonstrada em resposta a estrógeno exógeno, progesterona e gravidez, em um modelo utilizando ratos transgênicos HPVI $8^{35}$. Finalmente, hormônios esteróides, particularmente a progesterona, mostraram-se capazes de inibir a transativação transcricional de genes mediada por p53 envolvidos na interrupção do ciclo celular e apoptose ${ }^{54}$, bem como a expressão de antígenos principais de histocompatibilidade de classe I e $\|^{3,54}$.

Figura 2 - Interação molecular entre hormônios esteróides e elementos do HPV

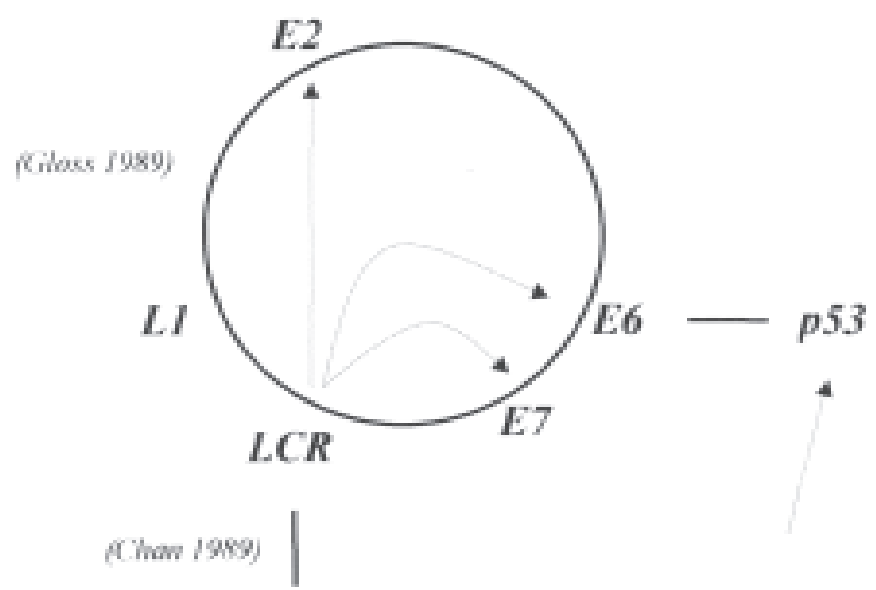

CiI ROCONTNCONDES PROGESTEROVA ESTRLCIENO

Airiaf (993)

(Mirnawi Jas9j
Estes dados experimentais, adicionados a observações epidemiológicas ${ }^{7,921}$ e clíni$\operatorname{cas}^{25,24,19,41,15}$, apontam para o uso de contraceptivos como um fator de risco no desenvolvimento do câncercervical. No entanto, alguns estudos recentes têm demonstrado ausência de associação entre 0 uso de contraceptivos orais e o $\mathrm{NIC}^{10,44}$.

O presente artigo de revisão colocou em foco os fatores não virais na patogênese do carcinomaescamosocervical. Verificou-se que estes fatores interagem em menor ou maior intensidade com as oncoproteínas e outros elementos do HPV, porém todos parecem relacionar-se com o vírus de forma a potencializar sua ação na célula hospedeira e facilitar o desenvolvimento dos processos de imortalização e carcinogênese. A participação exata de cada um dos fatores aqui abordados, quais os elementos essenciais para o desencadeamento do processo carcinogênico, e como isto ocorre in vivo, ainda são pontos a serem esclarecidos posteriormente.

\section{SUMMARY}

\section{HPV COFACTORS IN CERVICAL CARCl- NOGENESIS}

Human papillomavirus (HPV) plays a central rule in uterine cervix carcinogenesis. Other factors direct or indirectly influence the installation of this mechanism in cervical squamous epithelium. Investigations regarding mechanisms of interaction of these factors with viral elements are found in the literature of the last 20 years. The present review article discusses possible co-factors of HPV in the genesis of the squamous carcinoma of uterine cervix, taking into account only the factors whose association with the virus or cervical cancer has been documented by experimental studies, and not based just on clinical or epidemiological data. Among the approached parameters are immunological factors (local and humoral immune response), the association with Acquired Immune Deficiency Syn- 


\section{CO-FATORES DO HPV}

drome, genetic factors as protein p53 polymorphism, tabagism and the use of oral contraceptives. All these factors interact in variable intensity with oncoproteins and other HPV elements, increasing and facilitating the virus action in host cells, leading to the development of immortalization and carcinogenesis. [Rev Assoc Med Bras 2002; 48(I): 73-8]

KeY words: Human papillomavirus. Cervical cancer. Immunodepression. Tobacco. Oral contraceptives.

\section{REFERÊNCIAS}

I. Agorastos, T; Lambropoulos, A.F.; Constantinidis, T.C.; Kotsis, A.; Bontis, J.N. P53 codon 72 polymorphism and risk of intraepithelial and invasive cervical neoplasia in Greek women. Eur. J Cancer Prev., 9:1 I38, 2000.

2. Anton M, Horky M, Blaha O.The role of tumour suppressors and viral oncoproteins in cervicalcarcinogenesis.Ceska Gynekol.,65 (4):275-8, 2000.

3. Bartholomew, I.S.; Glenville, S.; Sarkar, Burt. D.J.; Stanley, M.A.; Ruiz- Cabello, F.; Chengang, J.; Garrido, F.; Stern, P.L. Integation of high-risk human papillomavirus DNA is linked to the down regulation of class I human leucocyte antigens by steroid hormones in cervical tumor cells. Cancer Res., 57(5):937-42, mar 1997.

4. Barton, S.E.; Hollingworth, A.; Maddox, P.H.; Edwards, R.; Cuzick, J.; McCance, D.J.; Jenkins, D.; Singer, A. Possible cdeactors in the etiology de cervical intraepithelial neoplasia. An immunopathologic study. J Reprod Med., 34(9):613-6, 1989.

5. Breirtburd, F. \& Cousrsaget, P. Human papillomavirus vaccines. Semin Cancer Biol., 9(6):431-44, 1999.

6. Brinck U, Jakob C, Bau O, Fuzesi L.Papillary squamous cell carcinoma of the uterine cervix: report of three cases and a review of its classification. Int J Gynecol Pathol., Jul; I 9(3):23I-5. 2000.

7. Brinton, L.A.; Reeves, W.C.; Brenes, M.M.; Herrero, R.; De Brinton, R.C.; Gaitan, F.T.; Garcia, M.; Ralws,W.E. Parity a risk for factor of cervical cancer. Am J Epidemiol., 130:486-496, 1989.

8. Brinton,L.A., et al. Risck fator for cervical cancer by histology. Gynaecol Oncol., $51(3): 301-6, \quad 1993$.

9. Brisson, J.; Morin, C.; Fortieri., M.; Roy, M.; Bouchard, C.; Lecler, J.; Christen, A.; Gui- mont, C.; Penault, F.; Meisels A. Risk factors for cervical cancer intraepithelial neoplasia: differences between low- and high-grade lesions. Am J Epidemiol., |40(8):700-10, 1994.

10. Cavalcanti SM, Zardo LG, Passos MR, Oliveira LH. Epidemiological aspects of human papillomavirus infection and cervical cancer in Brazil. J infect., 40(I):80-7, 2000.

I I. Chan, W.K.; Klock, G.; Bernard, H.U. Progesterone and glucocorticoid response elements occur in the long control regions of several human papillomaviruses involved in anogenital neoplasia. J Virol., 63:326I-269, 1989.

12. Cramer, D.W. \& Cutler, S.J. Incidence and histopathology of the malignancies of the female genital organs in the United States. Am J Obstet Gynecol., I |8:443-60, 1974.

13.Crespi, C.L.; Penman, B.W.; Gelboin, H.V.; Gonzales, F.J. A tobacco smoke-derived nitrosamine, 4-(methylnitrosamino)-I-(3pyridyl)-I-butanone, is activated by multiple human cytochrome P450s including the polymorphic human cytochrome P4502D6. Carcinogenesis., 12: |197-1201, 1991.

|4. Duensing S, Lee LY, Duensing A, basile J, Piboonniyom SO, Gonzalez S, Crum CP, Munger K. The human papillomavirus type E6 and E7 oncoproteins cooperate to induce mitotic defects and genomic instability by uncoupling centrosome duplication from the cell division cicle. Proc. Natl Acad Sci USA 2000; I5:epubahead of print.

15. Fife, K.; Katz, B.; Roush, J.; Handy, V.; Brown, D.; Hansell, R. Cancer associated human papillomavirus types are selectively increased in the cervix of women in the first semester of pregnancy. Am J Obstet Gynecol., 174: |487-93, 1996.

16. Franco, E.L; Duarte-Franco, E.; Ferenczy, A.Cervical cancer: epidemiology, prevention and the role of human papillomavirus infection. CMAJ., 164(7):1017-25, 2001.

17. Giannoudis A, Graham DA, Southern AS, Herrington CS. P53 codon 72 ARG/PRO plymorphism is not related to HPV type or lesion grade in low and high-grade squamous intra-epithelial lesions and invasive squamous carcinoma of the cervix. Int. J Canver., 24:66-9, 1999.

18.Gissmann L. Immunologic responses to human papillomavirus infection. In: Lorincz AT e Reid $\mathrm{R}$ (guest eds), Masse $\mathrm{S}$ (ed). Obstetrics e Gynecology Clinics of North America - Human Papillomavirus I. 1996; 23(3):625-639.

19. Gitsch, G.; Kainz, C.; Studinicka, M.; Reinthaller, A.; Tatra, G.; Breitenecker, G. Oral contaceptives and human papillomavirus infection in cervical intraepithelial neoplasia. Arch Gynecol., 252(I):25-30, 1992.

20. Gloss, B.; Bernard, H.U.; Seedorf, K.; Klock, $\mathrm{G}$. The upstream regulatiory region of the human papillomavirus 16 contains an E2 protein-indepedendent enhancer wich is is specific for cervical carcinoma cells and regulated by glucocorticoid hormones. EMBO J., 6(12):3735-43, 1987.

2 I. Gram, I.T.; Austin, H.; Stalberg, H. Cigarette smoking and the incidence of cervical itraepithelial neoplasia, grade III. And cancer of the cervix uteri. Am J Epidemiol., I35(4):34 I-6, 1992.

22. Haverkos, H.; Rohrer, M.; Pickworth, W. The cause of invasive cervical cancer could be multifactorial. Biomed Pharmacother., $54(1): 54-9, \quad 2000$

23. Hellberg, D.; Nilsson, S. Haley, N.J.; Hdefman, D.; Wynder, E. Smoking and cervical intraepithelial neoplasia: nicotine e cotidine in serum e cervical mucus in smokers e nonsmokers. Am J Obstet Gynecol., I58 (4): 910-3, 1988.

24. Herrero, R.; Brinton, L.A.; Reeves, W.C.; Brenes, M.M.; de Brinton, R.C.; Teonorio, F.; Gatain, E. Injectable contraceptive and risk of invasive cervical cancer: Evidence of an association. Int J Cancer.,45( I):5-7, 1990.

25. Hildestein, A.; Reeves, W.C.; Brenes, De La Guardia, M.E.; Godoy, J.; Rawls, W.E.; Association of oral contraceptive use and human papillomaviruses in invasive cervical cancers. Int / Cancer., 45(5):860-864, 1990.

26. Jochmus, I.; Schafger, K.; Faath,S.; Muller, M.; Gissman, L. Chimeric virus-like particles of the human papillomavirus type 16(HPV 16) as a prophylatic and therapeutic vaccine. Arch Med Res., 30(4):269-74, 1999.

27. Johnson, J.D.; Houchens, D.; Kluwe, W.M.; Craig, D.K.; Fisher, G.L. Effects of the mainstream and environmental tobacco smoke on the immune system in animals e humans: a review. Critical Reviews in Toxicology., 20 (5): 369-390, 1990.

28. Kjellberg, L.; Hallmans, G.; Ahren, A.M.; Johansson, R.; Bergman, F.; Wadell, G.; Angstrom, T.; Dillner, J. Smoking, diet, pregnancy and oral contraceptive use as risk factors for cervical intra-epithelial neoplasia in relation to human papillomavirus infection. Br J Câncer.,82(7): | 332-8, 2000.

29. Koskella, P. et al. Chlamydia Trachomatis infection as a risck factor for invasivecervical câncer. Int J Cancer., 85(I): 35-9,2000.

30. Ling M.; Kanayama, M.; Roden, R.; Wu, T.C. Preventive and therapeutic vaccines for human papillomavirus-associated cervical cancers. J Biomed Sci.,7(5):34l-56, 2000.

31. Lo, K,W.; Mok, C.H.; Chung, G.; Huang, D.P.; Wong, F.; Chan, M.; Lee, J.C.; Tsao, S.W. Presence of p53 mutation in human cervical carcinomas associated with HPV-33 infection. Anticancer Res, 1992; 12:1989-94.

32. Madeleine, M.M., et al. The p53 Arg72 Pro polymorphism, human papillomavirus, and invasive squamous cell cervical câncer. Cancer Epidemiol Biomarkers Prev., 9(2):225-7, 2000. 
33. Markowska, J. et al. The role of Chlamydia trachomatis infection in cervical cancer development. Eur J Gynaecol Oncol., 20(2): |44-6, 1999

34. McGlennen, R.C. Human papillomavirus oncogenesis. Clin Lab Med.,20(2):383-406, 2000.

35. Michelin, D.; Gissman, L.; Street, D; Potkul, R.K.; Fisher, S.; Kaufmann, A.M.; Qiao, L.; Schreckenberger, C. Regulation of human Papillomavirus type 18 in vivo: effects of estrogen and progesterone in transgenic mice. Gynecol Oncol., 66(2):202-8, 1997.

36. Milde-Langosch K, Riethdorf S, Loning T. Association of human papillomavirus infection with carcinoma of the cervix uteri and its precursor lesions: theoretical and practical implications. Virchows Arch., 437(3):227-33, 2000.

37. Mitrani-Rosenbaum, S.; Tsvieli, R.; TurKaspa: R. Oestrogen stimulates differential transcription of human papillomavirus type 16 in SiHa cervical carcinoma cells. / Gen Virol., 70:2227-2232, 1989

38. Mittal, R.; Tsutsumi, K.; Pater, A.; Pater, M. Human papillomavirus type 16 expression in cervical keratinocytes: Role of progesterone and glucocorticoid hormones. Obstet Gynecol., 81:5-12,1993.

39. Monsonego, J.; Magdelenat, $H_{\text {. }}$; Catalan, F. Coscas, Y.; Zerat, L.; Sastre, X. Estrogen and progesterone receptors in cervical human papillomavirus related lesions. Int J Cancer., 48(4):533-9, | 199 |.

40. Nuovo, G.J. The role of human papillomavirus in gynecological diseases. Crit Rev Clin Lab Sci., 37(3): 183-215, 2000.

4l. Pater, M.M.; Mittal, R.: Pater, A. Role of steroid hormones in potentating transformation of cervical cells by human papillomaviruses. Trends Microbiol., 2(7):229-235, 1994

42. Pinto, A. P., Crum, C.P. Natural history of cervical neoplasia: defining progression and its consequence. Clin Obstet Gynecol., 43(2): 352-62, 2000.
43. Poppe, W.A.; Drijkoningen, M.; Ide, P.S.; Laweryns, J.M.; Van Assche, F.A. Langerhans' cells and $\mathrm{LI}$ antigen expression in normal e abnormal squamous epithelium of the cervical transformation zone. Gynecol Obstet Invest, 4I(3):207-13, 1996.

44. Salazar, E.L.; Contreras, H.; Gonzalez, J.L. Effect oral contraceptive administration on the frequency of estrogen receptor and cervical intraepithelial neoplasia. Ginecol Obstet Mex., 68:160-4, 2000.

45. Silva, D.M., et al. Cervical cancer vaccines: emerging concepts and developments. J Cell Physiol.,. I86(2):I69-82, 200I.

46. Simons, A.M. Phillips, D.H.; Coleman, D.V. Damage to DNA in cervical epithelium related to smoking tobacco. $\mathrm{Br}$ Med J., 306:|444-|448, 1993.

47. Spandidos, D.A. Molecular basis of gynecological cancer. Ann N Y Acad Sci., 900:5664, 2000.

48. Stanley, M.A.; Coleman, N.; Chambers, M. The host response to lesions induced by human papillomavirus. In: Mindel A (ed). Genital Warts e Human Papillomavirus Infection. London:Edward Arnold, 1994. p. 21-44. 127.

49. Takehara, K. Local immune responses in uterine cervical carcinogenesis. Nippon Sanka Fujinka Gakkai Zasshi., 48(I I):1063-70, 1996.

50. Tay, S.K.; Jenkins, D.; Maddox, P.; Campion, M.; Singer, A. Subpopulations of the Langerhans' cells in cervical neoplasia. $\mathrm{Br}$ J Obstet Gynaecol., 94:10-15, 1987.

51. Van Duin, M.; Snijders, P.|.; Vossen, M.T. Klaassen, E.; Voohorst, F.; Verhieijen, R.H. Helmerhorst, T.J.; Meijer, C.J.; Walboomers, J.M. J Gen Virol ., 81:317-25, 2000.

52. Villa, L.L.; Human papilo mairuses and cervical cancer. Adv Cancer Res., v. 7I, p. $32|-4|, \quad 1997$.
53. Vinatier. D.; Cosson, M.; Dufour, P. Prophylactic and therapeutic vaccine therapy in papillomavirus infections. Gynecol Obstet Fertil., 28(5):370-84, 2000

54. Von Knebel Doberitz, M.; Spitkovasky, D.; Ridder, R. Interections between steroid hormones and viral oncogenes in the pathogenesis of cervical cancer. Verh Dtsch Ges Pathol., 81:233-9, 1997

55. Walboomers, J.M., et al..Human papillomavirus and the development of cervical cancer: concept of carcinogenesis. Ned Tijdschr Geneeskd., |44(35): |67|-4, 2000.

56.Warwick, A.P.; Redman, C.W.; Jones, P.W.; Fryer, AA.; Gilford, J.; Alldersea, J.; Strange, R.C. Progression de cervical intraepithelial neoplasia to cervical cancer: interactions de cytochrome P450 CYP2D6 EM e glutathione s-transferase GSTMI null genotypes e cigarette smoking. Br.J.Cancer., 70(4):704-8, 1994.

57. Warwick, A.; Sarhanis, P.; Redman, C.; Pemble, S.; Taylor, J.B.; Ketterer, B.; Jones, P.: Alldersea, J.; Gilford, J.; Yengi, L.; et al. Theta class glutathione S-transferase GSTTI genotypes e susceptibility to cervical neoplasia: interactions with GSTMI, CYP2D6 e smoking. Carcinogenesis., I 5( I 2):284 I-5, 1994

58. Wright, T.C. Sun XW Anogenital papillomavirus infection and neoplasia in immunodeficient women. In: Lorincz AT, Reid R (guest eds), Baniewicz C (ed). Obstetrics e Gynecology Clinics de North America Human Papillomavirus II. WB Saunders Company, 23(4):861-893, 1996.

59. Zehle I, Voglino G, Wilander E, genta $F$, Tommasino M. Codon 72 polymorphism of p53 and its association with cervical cancer. Lancet., 17:218-9, 1999.

\section{OPINIÃO DO LEITOR}

A nova Ramb abre espaço para o leitor na seção "Correspondências". Envie sua opinião ou sugestão sobre a revista ou artigos de seu interesse. Participe também enviando suas dúvidas ou sugestões sobre condutas práticas para a seção "À beira do leito". Sua colaboração é muito importante para aprimorarmos o nosso veículo de comunicação. Rua São Carlos do Pinhal, 324 Cep: 01333-903 - São Paulo - SP - E-mail:ramb@amb.org.br 